\title{
Focal therapy for prostate cancer: the technical challenges
}

\author{
Prof. Annette Haworth, BSc, MSc, PhD, FACPSEM!',2, Scott Williams, BSc, MBBS, MD, FRANZCR2,3 \\ ISchool of Physics, University of Sydney, Camperdown, NSW, ${ }^{2}$ Sir Peter MacCallum Department of Oncology. University of Melbourne, Melbourne, \\ Vic, ${ }^{3}$ Department of Radiation Oncology, Peter MacCallum Cancer Centre, Vic, Australia
}

\begin{abstract}
Focal therapy for prostate cancer has been proposed as an alternative treatment to whole gland therapy, offering the opportunity for tumor dose escalation and/or reduced toxicity. Brachytherapy, either low-dose-rate or high-doserate, provides an ideal approach, offering both precision in dose delivery and opportunity for a highly conformal, non-uniform dose distribution. Whilst multiple consensus documents have published clinical guidelines for patient selection, there are insufficient data to provide clear guidelines on target volume delineation, treatment planning margins, treatment planning approaches, and many other technical issues that should be considered before implementing a focal brachytherapy program. Without consensus guidelines, there is the potential for a diversity of practices to develop, leading to challenges in interpreting outcome data from multiple centers. This article provides an overview of the technical considerations for the implementation of a clinical service, and discusses related topics that should be considered in the design of clinical trials to ensure precise and accurate methods are applied for focal brachytherapy treatments.

Key words: brachytherapy, focal therapy, prostate cancer.

\section{Purpose}

Focal therapy has been proposed as the next major change in the way prostate cancer is treated; with only sub-regions of the prostate receiving high doses of radiation there is the potential for significant reductions in treatment related toxicity compared with conventional, whole gland treatments [1]. There is also the opportunity to escalate the dose to sub-regions of the gland, offering the potential for increased tumor control rates. Despite the potential advantages of focal therapy and demand from patients for improved treatment options, it is infrequently practiced. In this article, we will review current practices and suggest ways focal treatments could be introduced into the clinic.

The use of active surveillance in low and low-intermediate risk group patients has been recommended widely, citing the concern for undesirable side effects from treatment in patients at low-risk of death due to their disease [2]. However, the selection criteria for active surveillance remain controversial and unsuitable for anxious or non-compliant patients, and so there exists a need to develop treatment techniques that are cost effective, clinically effective, and with minimal toxicity. Focal therapy for prostate cancer has been the subject of discussion within multiple consensus groups $[3,4,5,6]$.
Each of these groups have provided recommendations for patient selection and, whilst it has been estimated that up to $75 \%$ of all low-risk prostate cancer patients may benefit from some form of focal therapy [6], to date only a small number of studies have reported clinical outcomes [7,8,9]. Despite the potential advantages of sparing normal tissue and lack of evidence for clinical effectiveness, we suggest there are likely to be multiple technical challenges and uncertainties that have limited widespread adoption of focal brachytherapy. Within this article, we will explore some of the technical challenges of introducing a focal brachytherapy program, and discuss the advantages and disadvantages of the various approaches that have been reported in the literature. Non-brachytherapy approaches to focal therapy are beyond the scope of this article. Similarly, we have not included a full discussion on "focal boost" strategies $[10,11,12,13,14]$, though many of the techniques we describe could apply to this treatment approach. The focus of this paper is to demonstrate how advanced technology may be exploited in developing a scientific approach to focal brachytherapy that will not only provide high quality treatments, but also provide a guide for the development of focal brachytherapy trials to determine the optimal approach to treatment. 


\section{Low-dose-rate or high-dose-rate brachytherapy?}

Whole gland, ultrasound-guided treatment using low-dose-rate (LDR) brachytherapy has been in routine clinical practice for well over twenty years, with many studies confirming high rates of tumor control in low-risk prostate cancer patients [15]. Whilst rectal side effects and erectile dysfunction rates are favorable compared with surgery and external beam radiotherapy, acute urinary bother is frequently reported and considered the major disadvantage of this treatment approach [16]. To overcome this limitation, focal LDR brachytherapy has been proposed as an option for appropriately selected patients [3]. Selection of isotope may depend on considerations of underdosing due the effects of edema, and overdosing the urethra due to seed migration [17]. However, in the few clinical studies reported, ${ }^{125} \mathrm{I}$ is most commonly used in LDR focal applications [9].

To date, few studies report the results of clinical outcomes using ${ }^{125} \mathrm{I}$ focal brachytherapy. Cosset et al., in a study of 21 patients, reported focal brachytherapy is feasible with little acute toxicity [7]. The target volume selected for irradiation was defined on mpMRI with a "rather large safety margin" such that approximately one-third of the entire prostate received 145 Gy. With a median follow-up of less than 18 months, it is too early to assess tumor control and long-term toxicity. Nguyen et al. reported the results of 318 patients with cT1c disease that received $137 \mathrm{~Gy}{ }^{125}$ I brachytherapy to the peripheral zone, noting that there is a low-risk of tumor foci in the anterior base, and that implanting seeds into this region has been associated with obstructive symptoms [8]. With a median follow-up of 5.1 years, 17 patients had biopsy proven local recurrence. In the case of low-risk disease (initial prostate specific antigen $[\mathrm{PSA}]<10 \mathrm{ng} / \mathrm{ml}$ and Gleason score $3+3$ ), biochemical control rates at 5 and 8 years were $95.6 \%$ and $90 \%$, respectively. However, in the case of the intermediate risk patients (PSA 10.1 to $15 \mathrm{ng} / \mathrm{ml}$ and Gleason score $3+4)$, biochemical control rates were $73 \%$ and $66.4 \%$ at 5 and 8 years, respectively, and hence these authors suggested that the peripheral gland approach may not be appropriate in these patients.

In the case of high-dose-rate (HDR) brachytherapy, whilst there are a number of planning studies exploring focal monotherapy treatment approaches, the results of clinical trials are yet to be reported $[11,12,18]$. The low $\alpha / \beta$ ratio for prostate cancer suggests HDR may be advantageous compared with LDR brachytherapy [19] in addition to the ability to optimally sculpt the radiation dose and achieve consistent implant quality [20].

\section{Target delineation}

Several approaches to target volume delineation have been proposed. The consensus group led by Langley et al., using an LDR approach presented three scenarios, which can be summarized as: 1) ultra-focal therapy, whereby the volume of tissue treated is confined to the region containing the cancer; 2 ) focal therapy (also known as hemi-gland therapy), whereby the contralateral gland is spared; 3) focused therapy, where the ablative dose is confined to the tumor bearing region, with the remaining gland receiving a lower dose [3]. Other approaches using a geometric (rather than anatomical) subdivision have been described, for example the HDR planning study of Mason et al. used a sector approach to boost sub-volumes [21]. The definition of target volume for ultra-focal and/ or focused therapy is controversial [22]. Prostate cancer is typically multifocal with the dominant or index lesion typically defined as the tumor with the largest volume. Whilst there is some evidence to suggest that the characteristics of the index lesion will predict clinical outcome $[23,24]$, smaller non-index tumors, not easily detected on imaging or biopsy, may contain poorly differentiated elements, which may ultimately determine the risk of metastases [25]. So, while the ultra-focal approach may provide maximal healthy tissue sparing, this approach needs to be used with caution, and every effort made to rule out significant cancers in the smaller lesions. The "focused" approach takes into account the multi-focal nature of the disease, with the lower dose taking care of smaller tumor volumes not easily detected on biopsy or multiparametric magnetic resonance imaging (mpMRI).

There appears to be a consensus that mpMRI plays a role in focal therapy [26]. Firstly, mpMRI may be used to guide biopsy with several authors indicating mpMRI guided biopsy has a high negative predictive value [27] and may improve the detection of clinically significant cancers [28]. Similarly, mpMRI is the most commonly suggested method for defining gross tumor volume (GTV) due to its high sensitivity and specificity [29]. Several studies have attempted to validate tumor volume delineation on mpMRI using pathology as the 'ground truth' [30,31,32]. Such studies are challenging and require sophisticated methods for co-registration of histology and mpMRI [31]. Furthermore, machine learning techniques have been used to develop predictive models to produce accurate and automated methods for tumor delineation [30,33]. To account for uncertainties in defining the extent of the disease, an additional margin is typically applied to create the clinical target volume (CTV). Additional margins are required to account for a large range of uncertainties, such as those from resolution of the imaging data, image co-registration, and treatment delivery uncertainties. Each of these uncertainties must be quantified for the specific imaging and treatment delivery approaches. A full review of these uncertainties is beyond the scope of this paper, however, the use of statistical methods (probability maps) may offer a flexible approach to incorporation of these uncertainties [32,34].

Multiparametric MRI is generally recognized as a useful tool in the staging and grading of newly diagnosed prostate cancer, and the PI-RADS system provides recommendations for reporting clinically significant cancers [35]. Historically, positron emission tomography (PET) imaging has largely been used in the diagnosis and management of systemic disease [36] and therefore, in the context of focal therapy, only useful in identifying patients unsuitable for highly localized (focal) treatment. In recent years, there has been a growing interest in the use of ${ }^{68} \mathrm{Ga}$-PSMA PET in combination with mpMRI in the diagnosis and staging of primary and recurrent cancer. 
Several small studies have demonstrated that ${ }^{68} \mathrm{Ga}-\mathrm{PSMA}$ PET was more specific for prostate cancer than mpMRI alone, when compared with whole mount histology $[37,38,39,40]$. However, these validation studies typically divided the prostate into sextants for analysis of sensitivity and specificity. This approach may be adequate for a hemi-gland or sector approach, but in the case of focal or focused approaches, mpMRI will remain essential for GTV delineation with ${ }^{68} \mathrm{Ga}$-PSMA PET playing an important role in confirming selection of suspicious regions on mpMRI for treatment. Furthermore, we believe ${ }^{68} \mathrm{Ga}-\mathrm{PSMA}$ PET will play a significant role in identifying patients with true locally recurrent prostate cancer and low PSA who may be suitable for focal salvage therapy $[41,42,43]$.

Precise delineation of tumor volumes should provide maximal sparing of organs at risk (urethra, bladder, neurovascular bundles, and rectum). However, Mason et al., using an HDR focal boost (with whole gland EBRT) compared a target volume defined on mpMRI approach with a sector approach, which involved dividing the base, mid-gland, and apex into quadrants. Minimal differences were seen between conventional and boost plans [21]. In this study, the target volumes were defined with a generous $4.5 \mathrm{~mm}$ margin around the tumor to take into account delineation and mpMRI/TRUS co-registration uncertainties - these uncertainties may have been amplified by the androgen deprivation used in this study, with resultant smaller prostate volumes, and reduced contrast between benign and cancerous prostate tissue [44].

\section{Treatment planning}

In the case of LDR focal therapy, it is likely that a combination of pre-planning and real-time adaptive planning would be employed in the early stages of developing a focal program. Pre-planning provides time for the team to carefully consider treatment approaches and planning objectives prior to the implant procedure [3]. During the procedure, seed placement can be accurately monitored and additional seeds implanted if unexpected, significant seed migration or edema is observed. As noted by Al-Qaisieh et al., focal plans are more sensitive to seed displacement errors [45]. We suggest that ideally seed placement should occur using the planning MRI images co-registered with the ultrasound images [3]. However, the limitations of rigid and deformable image registration techniques must be carefully considered with this approach (see treatment delivery below).

The consensus group suggested using stranded seeds on the periphery of the target volume due to migration, and loose seeds around the urethra to provide greater flexibility [3]; however, the selection of seed type may depend on implant technique $[46,47]$. The consensus group recommended dose constraints to the urethra and rectum follow current whole-gland limits [3,48,49]; however, we suggest this is a topic for further investigation, and that dose to other organs at risk such as the bladder, penile bulb, and neurovascular bundles be recorded to further improve our understanding of the dose-response relationship.
Regarding focal HDR, Mason et al. reported treatment planning approaches that incorporated an HDR boost to prostate sub-volumes in addition to external beam radiotherapy $[10,21]$. Dankulchai et al. reported the results of an HDR monotherapy planning study that incorporated a (HDR) focal boost [11]. In the latter study, sub-volumes in 16 patients, each with 1-3 boost target volumes, were defined using mpMRI. The sub-volumes were delineated by an experienced oncologist, and expanded by a $3 \mathrm{~mm}$ margin to achieve a $10 \%$ boost dose using a single fraction, with a whole gland dose prescription of $19 \mathrm{~Gy}$. Using a $5 \mathrm{~mm}$ needle spacing (rather than the standard $10 \mathrm{~mm}$ ) through the sub-volumes, it was found to improve the likelihood of meeting planning objectives, including: urethral $\mathrm{D}_{30}$ (dose received by $30 \%$ of the urethral volume), $\mathrm{D}_{10}, \mathrm{~V}_{150 \%}$ (the percentage of the volume receiving $150 \%$ of the prescribed dose or more) less than $20.8 \mathrm{~Gy}, 22 \mathrm{~Gy}$, and $0.01 \mathrm{cc}$ (minimum dose to the most exposed $0.01 \mathrm{cc}$ ), respectively. This $5 \mathrm{~mm}$ spacing also improved the conformity of the dose to the defined sub-volumes compared with standard spacing. This study clearly demonstrates a feasible approach; however, these authors acknowledge that delineation of the dominant region remains controversial. In addition, mature clinical data for this approach is not yet available and that the optimal dose planning objectives are, as yet, not confirmed.

\section{Dose prescription and fractionation}

For LDR focal therapy, the consensus group suggested further modelling is required for prescription dose recommendations and will depend on the approach to target definition. For example, 145 Gy to the index lesion could be prescribed with a lower dose to low-risk areas [3]. Clearly, this is an area of great uncertainty, and we suggest that novel approaches using biological methods for dose prescription be considered in future studies [50]. This approach will require inverse optimization techniques using biological objective functions to aid the treatment planning process, as forward planning approaches would be more challenging compared with conventional dose planning methods [51].

Similarly, HDR dose prescription methods are based on our experience with whole-gland approaches, though with variations in fractionation schedules and planning objectives (tumor dose boost vs. OAR sparing), we believe there is an even more urgent need for studies to address the optimal approach to focal HDR brachytherapy [9]. Clinical evidence for optimal dose fractionation and scheduling with EBRT in the focal setting is also currently lacking. Whilst we await the results of the FLAME [52] and Hypo-FLAME trials (www.clinicaltrials.gov), we suggest this be considered in future clinical and modelling studies.

\section{Treatment delivery}

Partial irradiation of the prostate requires precise delivery techniques to achieve optimal therapeutic gain. Real-time MRI guided treatment provides an ideal method for minimizing or accounting for dose delivery uncertainties [53]. However, availability of an MR unit is often 
limited for lengthy implant procedures. Hence, HDR and LDR brachytherapy techniques typically involve MR and ultrasound imaging in the treatment workflow, including at the time of biopsy and then at treatment delivery. A number of commercial systems exist to co-register MRI with ultrasound, however the uncertainty in the co-registration process is typically not well understood [54]. Rigid registration of MRI data (obtained with the patient in the supine position) with the ultrasound data (obtained with the patient in the lithotomy position) may not provide sufficient accuracy for a focused or ultrafocused approach. Using deformable image registration (DIR) techniques may provide a more accurate solution $[55,56,57]$, but validation of the registration during the implant procedure may be challenging for a clinical team unfamiliar with the limitations of DIR algorithms.

Robotic systems using 3D ultrasound offer the possibility of tracking prostate motion during the brachytherapy procedure with an accuracy of $1.1 \mathrm{~mm}$ [58]. These systems, however, still rely on pre-operative MRI as ultrasound cannot provide sufficient accuracy for tumor delineation.

Uncertainties in treatment delivery such as seed migration (for LDR) or catheter movement between treatment planning and treatment delivery (for HDR), may be accounted for in the treatment margin. In the case of LDR, the margin may be determined by comparing pre- and post-implant seed positions [59] or through mathematical modelling of the dose rate function [60].

\section{Post-implant evaluation and clinical follow-up}

Post-implant dosimetry for LDR focal therapy was recommended either within 24-hours of surgery or at 4 -weeks post-implant by the consensus group [3]. Clearly, this is particularly important in this group of patients where treatment plans may be less robust to seed movement. However, as we develop clinical experience with focal therapy, it will also provide an opportunity to improve our understanding of the dose-response relationship, so that better estimates of dose constraints can be derived. Tong et al. [17] in addition, recommended using the conventional whole gland dosimetry parameters for reporting implant quality and dose to organs at risk [49].

Reporting LDR implant quality in the whole gland approach has been controversial with several groups, noticing the standardly used $\mathrm{D}_{90}$ (dose to $90 \%$ of the prostate volume) parameter may be technique specific, and does not account for the spatial relationship of low-dose regions, and the likelihood of the presence of tumor foci being present in that region $[61,62,63]$. There is very limited clinical data supporting a dose-to-tumor relationship, and biochemical control in the focal setting and research in this area is urgently needed. This is complicated further by the fact that standard definitions for biochemical control [64] is unlikely to be appropriate when a reasonable (and variable) proportion of the prostate gland is left untreated [8]. Imaging in this scenario is likely to play a major role $[65,66,67]$ with several studies reporting the use of mpMRI in post treatment surveillance $[47,48]$. Currently, the optimal selection of imaging sequences for detection of recurrent prostate cancer is unclear with standard $\mathrm{T} 2 \mathrm{w}$ imaging sequences difficult to interpret post-irradiation $[68,69]$.

\section{Salvage therapy}

Whilst there is limited evidence for focal brachytherapy approaches in the definitive setting, there is even less evidence for salvage therapies. However, it is potentially in the salvage setting that novel treatment approaches are more urgently required as tumor control rates are suboptimal and toxicity significant compared to primary treatment options [69]. Indeed, a significant proportion of post-EBRT prostate biopsies demonstrate local residual or recurrent disease, which could be amenable to focal salvage therapies [69]. In a systematic review of salvage therapy options following post-irradiation biochemical failure, Nguyen et al. suggested that patients with low-risk disease at the time of local (only) failure may be suitable candidates for salvage therapy. However, post-salvage toxicity was reported to be considerable in some series and further research is required to identify post-salvage morbidity risk factors [70]. A small number of small series with short follow-up periods have reported favorable results in patients treated with a focal salvage approach using ${ }^{125}$ I seeds $[71,72,73]$. In a study by Kunogi et al. for example, 12 patients were treated using a focal approach with ${ }^{125}$ I seeds [73]. With a median follow-up time of 56 months, biochemical recurrence was reported in 2 patients and no Grade 3 GU/GI toxicities were reported, suggesting this approach warrants further investigation. Defining dose constraints for treatment planning will be particularly difficult in this group of patients and is likely to depend on the radiation dose delivered during the primary treatment (if applicable); we suggest that multiple anatomical structures be defined during the planning stages to build on the evidence for bladder and urethral constraints as reported by Peters et al. [74,75]. mpMRI is again likely to play an important role in guiding biopsies and defining volumes for treatment and post-treatment surveillance $[68,76]$.

\section{Discussion and future work}

Whilst the concept of focal brachytherapy has been discussed at many levels, there is not yet clear evidence for its safety and clinical efficacy. The small number of clinical trials that have provided early evidence for this efficacy, provide limited information on the technical details of the treatment planning and treatment delivery approaches, which may lead to some difficulties in defining the cause of treatment failures (e.g. geographic miss or insufficient dose), and how we may improve our approaches in the future. Additionally, the dose response to tumors and normal tissue treated using a focal approach is poorly understood.

There are however, many advances in our understanding of the role of mpMRI in diagnosis, treatment planning, and post-treatment surveillance that offer increased confidence in introducing focal brachytherapy into the clinic. In particular, we suggest that advances in quantitative imaging (radiomics) provides many opportunities to not 
only better identify and target high-risk volumes, but also determine a better understanding of the biology and heterogeneity of the tumor(s), providing an opportunity to customize dose prescriptions to the individual patient $[50,68,77]$. For example, identifying regions of hypoxia provides an opportunity to dose escalate to overcome radioresistance in these areas [78,79]. By using a voxelised approach to treatment planning and delivery, we have an opportunity to improve our understanding of the spatial relationship of dose to tumors and healthy tissue. Such an approach is demanding but not impossible, and by adopting this approach, we have the opportunity to ensure the success of future clinical studies.

Currently, there are just three registered trials recruiting patients for focal brachytherapy using LDR and two Phase II HDR trials for primary prostate cancer (https:/ / www.clinicaltrials.gov). There are however, a further six trials that are listed as "not yet recruiting", including two trials offering salvage focal brachytherapy. Whilst we eagerly await the outcomes of these trials, we strongly encourage the collection and reporting of both clinical and technical information, so that the evidence for the optimal treatment approach can be established [6].

\section{Conclusions}

This article has focused on the technical challenges of introducing a focal brachytherapy program, noting that many consensus reports provide detailed discussions on the clinical aspects. Focal brachytherapy has the potential to achieve significant gains in minimizing treatment related toxicity and increase tumor control, but is not yet widely practiced. Until clear evidence exists that focal therapy is safe and clinically effective, it should only be practiced in the context of a clinical trial. We have presented an overview of some of the technical challenges in introducing a focal program, and provide suggestions for a scientific approach to applying advanced technology to develop a precise and accurate method for focal brachytherapy treatments.

\section{Disclosure}

Authors report no conflict of interest.

\section{References}

1. Valerio M, Cerantola Y, Eggener SE et al. New and Established Technology in Focal Ablation of the Prostate: A Systematic Review. Eur Urol 2017; 71: 17-34.

2. van der Poel H, Klotz L, Andriole G et al. Role of active surveillance and focal therapy in low- and intermediate-risk prostate cancers. World J Urol 2015; 33: 907-916.

3. Langley S, Ahmed HU, Al-Qaisieh B et al. Report of a consensus meeting on focal low dose rate brachytherapy for prostate cancer. BJU Int 2012; 109 Suppl 1: 7-16.

4. Donaldson IA, Alonzi R, Barratt D et al. Focal Therapy: Patients, Interventions, and Outcomes - A Report from a Consensus Meeting. Eur Urol 2015; 67: 771-777.

5. van den Bos W, Muller BG, Ahmed H et al. Focal therapy in prostate cancer: international multidisciplinary consensus on trial design. Eur Urol 2014; 65: 1078-1083.

6. Ahmed HU, Berge V, Bottomley D et al. Can we deliver ran- domized trials of focal therapy in prostate cancer? Nat Rev Clin Oncol 2014; 11: 482-491.

7. Cosset JM, Cathelineau X, Wakil G et al. Focal brachytherapy for selected low-risk prostate cancers: a pilot study. Brachytherapy 2013; 12: 331-337.

8. Nguyen PL, Chen M, Zhang Y et al. Updated Results of Magnetic Resonance Imaging Guided Partial Prostate Brachytherapy for Favorable Risk Prostate Cancer: Implications for Focal Therapy. J Urol 2012; 188: 1151-1156.

9. Peach MS, Trifiletti DM, Libby B. Systematic Review of Focal Prostate Brachytherapy and the Future Implementation of Image-Guided Prostate HDR Brachytherapy Using MR-Ultrasound Fusion. Prostate Cancer 2016; 2016: 4754031.

10. Mason J, Al-Qaisieh B, Bownes P et al. Multi-parametric MRIguided focal tumor boost using HDR prostate brachytherapy: a feasibility study. Brachytherapy 2014; 13: 137-145.

11. Dankulchai P, Alonzi R, Lowe GJ et al. Optimal source distribution for focal boosts using high dose rate (HDR) brachytherapy alone in prostate cancer. Radiother Oncol 2014; 113: 121-125.

12. Kamrava M, Chung MP, Kayode $\mathrm{O}$ et al. Focal high-dose-rate brachytherapy: A dosimetric comparison of hemigland vs. conventional whole-gland treatment. Brachytherapy 2013; 5: 434-441.

13. Zaorsky NG, Doyle LA, Yamoah K et al. High dose rate brachytherapy boost for prostate cancer: a systematic review HDR-BT. Cancer Treat Rev 2014; 40: 414-425.

14. Gomez-Iturriaga A, Casquero F, Urresola A et al. Dose escalation to dominant intraprostatic lesions with MRI-transrectal ultrasound fusion High-Dose-Rate prostate brachytherapy. Prospective phase II trial. Radiother Oncol 2016; 119: 91-96.

15. Grimm PD, Blasko JC, Sylvester JE, et al. 10-year biochemical (prostate-specific antigen) control of prostate cancer with (125) I brachytherapy. Int I Radiat Oncol Biol Phys 2001; 51: 31-40.

16. Sanda MG, Dunn RL, Michalski J et al. Quality of Life and Satisfaction with Outcome among Prostate-Cancer Survivors. N Engl J Med 2008; 358: 1250-1261.

17. Tong WY, Cohen G, Yamada Y. Focal low-dose rate brachytherapy for the treatment of prostate cancer. Cancer Manag Res 2013; 5: 315-325.

18. Mason J, Al-Qaisieh B, Bownes P et al. Dosimetry modeling for focal high-dose-rate prostate brachytherapy. Brachytherapy 2014; 13: 611-617.

19. Duchesne GM, Peters LJ. What is the alpha/beta ratio for prostate cancer? Rationale for hypofractionated high-dose-rate brachytherapy. Int J Radiat Oncol Biol Phys 1999; 44: 747-748.

20. Morton G, Hoskin PJ. Brachytherapy: Current Status and Future Strategies - Can High Dose Rate Replace Low Dose Rate and External Beam Radiotherapy? Clin Oncol (R Coll Radiol) 2013; 25: 474-482.

21. Mason J, Bownes P, Carey B et al. Comparison of focal boost high dose rate prostate brachytherapy optimisation methods. Radiother Oncol 2015; 117: 521-524.

22. Murphy DG, Walton TJ, Connolly S, Costello AJ. Focal therapy for localised prostate cancer: are we asking the correct research questions? BJU Int 2012; 109: 1-3.

23. Villers A, McNeal JE, Freiha FS, Stamey TA. Multiple cancers in the prostate. Morphologic features of clinically recognized versus incidental tumors. Cancer 1992; 70: 2313-2318.

24. Wise AM, Stamey TA, McNeal JE, Clayton JL. Morphologic and clinical significance of multifocal prostate cancers in radical prostatectomy specimens. Urology 2002; 60: 264-269.

25. Andreoiu M, Cheng L. Multifocal prostate cancer: biologic, prognostic, and therapeutic implications. Hum Pathol 2010; 41: 781-793.

26. Tran M, Thompson J, Bohm M et al. Combination of multiparametric MRI and transperineal template-guided mapping biopsy of the prostate to identify candidates for hemi-ablative focal therapy. Urol Oncol 2016; 117: 48-54. 
27. Fütterer JJ, Briganti A, De Visschere P et al. Can Clinically Significant Prostate Cancer Be Detected with Multiparametric Magnetic Resonance Imaging? A Systematic Review of the Literature. Eur Urol 2015; 68: 1045-1053.

28. Ahmed HU, Bosaily AE, Brown LC et al. Diagnostic accuracy of multi-parametric MRI and TRUS biopsy in prostate cancer (PROMIS): a paired validating confirmatory study. Lancet 2017; 6736: 1-8.

29. van der Heide UA, Houweling AC, Groenendaal G et al. Functional MRI for radiotherapy dose painting. Magn Reson Imaging 2012; 30: 1216-1223.

30. Sun Y, Reynolds H, Wraith D et al. Predicting prostate tumour location from multiparametric MRI using Gaussian kernel support vector machines: a preliminary study. Australas Phys Eng Sci Med 2017; 40: 39-49.

31. Reynolds HM, Williams S, Zhang A et al. Development of a registration framework to validate MRI with histology for prostate focal therapy. Med Phys 2015; 42 : 7078.

32. Groenendaal G, Borren A, Moman MR et al. Pathologic validation of a model based on diffusion-weighted imaging and dynamic contrast-enhanced magnetic resonance imaging for tumor delineation in the prostate peripheral zone. Int J Radiat Oncol Biol Phys 2012; 82: e537-544.

33. Shah V, Turkbey B, Mani H et al. Decision support system for localizing prostate cancer based on multiparametric magnetic resonance imaging. Med Phys 2012; 39: 4093-4103.

34. Korporaal JG, van den Berg CA, Groenendaal G et al. The use of probability maps to deal with the uncertainties in prostate cancer delineation. Radiother Oncol 2010; 94: 168-172.

35. Weinreb JC, Barentsz JO, Choyke PL et al. PI-RADS Prostate Imaging - Reporting and Data System: 2015, Version 2. Eur Urol 2016; 69: 16-40.

36. Moghanaki D, Turkbey B, Vapiwala N et al. Advances in Prostate Cancer Magnetic Resonance Imaging and Positron Emission Tomography-Computed Tomography for Staging and Radiotherapy Treatment Planning. Sem Radiat Oncol 2017; 27: 21-33.

37. Rowe SP, Gage KL, Faraj SF et al. ${ }^{18}$ F-DCFBC PET/CT for PSMA-Based Detection and Characterization of Primary Prostate Cancer. J Nucl Med 2015; 56: 1003-1010.

38. Rhee H, Thomas P, Shepherd B et al. Prostate Specific Membrane Antigen Positron Emission Tomography May Improve the Diagnostic Accuracy of Multiparametric Magnetic Resonance Imaging in Localized Prostate Cancer. J Urol 2016; 196: 1261-1267.

39. Eiber M, Weirich G, Holzapfel K et al. Simultaneous 68Ga-PSMA HBED-CC PET/MRI Improves the Localization of Primary Prostate Cancer. Eur Urol 2016; 70: 829-836.

40. Zamboglou C, Drendel V, Jilg CA et al. Comparison of ${ }^{68} \mathrm{Ga}$ HBED-CC PSMA-PET/CT and multiparametric MRI for gross tumour volume detection in patients with primary prostate cancer based on slice by slice comparison with histopathology. Theranostics 2017; 7: 228-237.

41. Morigi JJ, Stricker PD, van Leeuwen PJ et al. Prospective Comparison of 18F-Fluoromethylcholine Versus 68Ga-PSMA PET/CT in Prostate Cancer Patients Who Have Rising PSA After Curative Treatment and Are Being Considered for Targeted Therapy. J Nucl Med 2015; 56: 1185-1190.

42. Maenhout M, van Vulpen M, Moerland M et al. Second salvage high-dose-rate brachytherapy for radiorecurrent prostate cancer. I Contemp Brachytherapy 2017; 9: 161-166.

43. Zamboglou C, Rischke HC, Meyer PT et al. Single fraction multimodal image guided focal salvage high-dose-rate brachytherapy for recurrent prostate cancer. J Contemp Brachytherapy 2016; 8: 241-248.

44. Padhani AR, MacVicar AD, Gapinski CJ et al. Effects of Androgen Deprivation on Prostatic Morphology and Vascular Permeability Evaluated with MR Imaging. Radiology 2001; 218: 365-374.
45. Al-Qaisieh B, Mason J, Bownes P et al. Dosimetry Modeling for Focal Low-Dose-Rate Prostate Brachytherapy. Int J Radiat Oncol Biol Phys 2015; 92: 787-793.

46. Hinnen KA, Moerland MA, Battermann JJ et al. Loose seeds versus stranded seeds in I-125 prostate brachytherapy: Differences in clinical outcome. Radiother Oncol 2010; 96: 30-33.

47. Saibishkumar EP, Borg J, Yeung I et al. Sequential Comparison of Seed Loss and Prostate Dosimetry of Stranded Seeds With Loose Seeds in 125I Permanent Implant for Low-Risk Prostate Cancer. Int J Radiat Oncol Biol Phys 2009; 73: 61-68.

48. Davis BJ, Horwitz EM, Lee WR et al. American Brachytherapy Society consensus guidelines for transrectal ultrasound-guided permanent prostate brachytherapy. Brachytherapy 2012; 11: 6-19.

49. Salembier C, Lavagnini P, Nickers P et al. Tumour and target volumes in permanent prostate brachytherapy: a supplement to the ESTRO/EAU/EORTC recommendations on prostate brachytherapy. Radiother Oncol 2007; 83: 3-10.

50. Haworth A, Williams S, Reynolds H et al. Validation of a radiobiological model for low-dose-rate prostate boost focal therapy treatment planning. Brachytherapy 2013; 12: 628-636.

51. Haworth A, Mears C, Betts JM et al. A radiobiology-based inverse treatment planning method for optimisation of permanent 1-125 prostate implants in focal brachytherapy. Phys Med Biol 2016; 61: 430-444.

52. Lips IM, van der Heide UA, Haustermans K et al. Single blind randomized phase III trial to investigate the benefit of a focal lesion ablative microboost in prostate cancer (FLAME-trial): study protocol for a randomized controlled trial. Trials 2011; 12: 255.

53. Borot de Battisti M, Maenhout M, Denis de Senneville B et al. An automated optimization tool for high-dose-rate (HDR) prostate brachytherapy with divergent needle pattern. Phys Med Biol 2015; 60: 7567-7583.

54. Logan JK, Rais-Bahrami S, Turkbey B et al. Current status of magnetic resonance imaging (MRI) and ultrasonography fusion software platforms for guidance of prostate biopsies. BJU Int 2014; 114: 641-652.

55. Mitra J, Marti R, Oliver A et al. Prostate multimodality image registration based on B-splines and quadrature local energy. Int J Comput Assist Radiol Surg 2012; 7: 445-454.

56. van de Ven WJ, Hu Y, Barentsz JO et al. Biomechanical modeling constrained surface-based image registration for prostate MR guided TRUS biopsy. Med Phys 2015; 42: 2470-2481.

57. Zhang S, Jiang S, Yang Z, Liu R. 2D Ultrasound and 3D MR Image Registration of the Prostate for Brachytherapy Surgical Navigation. Medicine (Baltimore) 2015; 94: e1643.

58. Hungr N, Baumann M, Long JA, Troccaz J. A 3-D ultrasound robotic prostate brachytherapy system with prostate motion tracking. IEEE Trans Robot 2012; 28: 1382-1397.

59. Polders DL, Steggerda M, van Herk M et al. Establishing implantation uncertainties for focal brachytherapy with I-125 seeds for the treatment of localized prostate cancer I-125 seeds for the treatment of localized prostate cancer. Acta Oncol (Madr) 2015; 54: 839-846.

60. Betts JM, Mears C, Reynolds HM et al. Optimised robust treatment plans for prostate cancer focal brachytherapy. Procedia Comput Sci 2015; 51: 914-923.

61. Haworth A, Ebert M, Waterhouse D et al. Assessment of I-125 prostate implants by tumor bioeffect. Int J Radiat Oncol Biol Phys 2004; 59: 1405-1413.

62. Spadinger I, Morris WJ, Keyes $M$ et al. Quadrant dosimetry as a predictor of biochemical relapse in 125I prostate brachytherapy. Brachytherapy 2011; 10: 87-97.

63. Morris WJ, Halperin R, Spadinger I. Point: the relationship between postimplant dose metrics and biochemical no evidence of disease following low dose rate prostate brachytherapy: is there an elephant in the room? Brachytherapy 2010; 9: 289-292. 
64. Roach M 3rd, Hanks G, Thames Jr. H et al. Defining biochemical failure following radiotherapy with or without hormonal therapy in men with clinically localized prostate cancer: recommendations of the RTOG-ASTRO Phoenix Consensus Conference. Int J Radiat Oncol Biol Phys 2006; 65: 965-974.

65. Muller BG, van den Bos W, Brausi M et al. Follow-up modalities in focal therapy for prostate cancer: results from a Delphi consensus project. World J Urol 2015; 33: 1503-1509.

66. Decker G, Mürtz P, Gieseke J et al. Intensity-modulated radiotherapy of the prostate: Dynamic ADC monitoring by DWI at 3.0 T. Radiother Oncol 2014; 113: 115-120.

67. De Visschere PJ, De Meerleer GO, Fütterer JJ et al. Role of MRI in follow-up after focal therapy for prostate carcinoma. AJR Am J Roentgenol 2010; 194: 1427-1433.

68. Barchetti F, Panebianco V. Multiparametric MRI for recurrent prostate cancer post radical prostatectomy and postradiation therapy. Biomed Res Int 2014; 2014: 316272.

69. Tetreault-Laflamme, A Crook J. Options for Salvage of Radiation Failures for Prostate Cancer. Semin Radiat Oncol 2016; 27: 67-78.

70. Nguyen PL, D'Amico AV, Lee AK, Suh WW. Patient selection, cancer control, and complications after salvage local therapy for postradiation prostate-specific antigen failure: a systematic review of the literature. Cancer 2007; 110: 1417-1428.

71. Hsu CC, Hsu H, Pickett B et al. Feasibility of MR imaging/ MR spectroscopy-planned focal partial salvage permanent prostate implant (PPI) for localized recurrence after initial PPI for prostate cancer. Int J Radiat Oncol Biol Phys 2013; 85: 370-377.

72. Peters M, Maenhout M, van der Voort van Zyp JR et al. Focal salvage iodine-125 brachytherapy for prostate cancer recurrences after primary radiotherapy: A retrospective study regarding toxicity biochemical outcome and quality of life. Radiother Oncol 2014; 112: 77-82.

73. Kunogi H, Wakumoto Y, Yamaguchi N et al. Focal partial salvage low-dose-rate brachytherapy for local recurrent prostate cancer after permanent prostate brachytherapy with a review of the literature. J Contemp Brachytherapy 2016; 8: 165-172.

74. Peters M, Hoekstra CJ, van der Voort van Zyp JR et al. Rectal dose constraints for salvage iodine-125 prostate brachytherapy. Brachytherapy 2016; 15: 85-93.

75. Peters M, van der Voort van Zyp JR, Hoekstra C et al. Urethral and bladder dosimetry of total and focal salvage Iodine-125 prostate brachytherapy: Late toxicity and dose constraints. Radiother Oncol 2015; 117: 262-269.

76. Ménard C, Iupati D, Publicover J et al. MR-guided prostate biopsy for planning of focal salvage after radiation therapy. Radiology 2015; 274: 181-191.

77. Jaffray DA, Chung C, Coolens C et al. Quantitative Imaging in Radiation Oncology: An Emerging Science and Clinical Service. Semin Radiat Oncol 2015; 25: 292-304.

78. Stoyanova R, Takhar M, Tschudi Y et al. Prostate cancer radiomics and the promise of radiogenomics. Transl Cancer Res 2016; 5: 432-447.

79. Gómez-Millán J, Lara MF, Correa GR et al. Advances in the treatment of prostate cancer with radiotherapy. Crit Rev Oncol Hematol 2015; 95: 144-153. 